\title{
30
}

\section{A QoS Configuration System for Distributed Applications}

\author{
Alan Smith, Andrew Grace \\ Distributed Systems Group, BT Laboratories, \\ Martlesham Heath, Ipswich, IP5 3RE, England \\ email: [asmith, apgrace] @jungle.bt.co.uk
}

\begin{abstract}
The range of distributed applications has increased dramatically over recent years, fuelled in particular by the growth of the Internet and other Local Area and Wide Area Networks. Such applications tend to require user level knowledge of low-level technical parameters and an appreciation of system heterogeneity issues, rather than simply stating what service they require.

In this short paper we describe a working system for configuring QoS for MBone conferencing applications. Here we use $Q 0 S$ profiles as a means of specifying resources and requirements.
\end{abstract}

Key words: QoS profiles, QoS resources, QoS requirements

\section{INTRODUCTION}

This paper describes a system for configuring distributed multimedia services running in a heterogeneous environment, based upon the principle of using declarative QoS profiles [1]. There are two basic classes of QoS profile used in our approach: QoS required and QoS provided. We address the QoS of a media-type in terms of the end system capabilities rather than characteristics of the mediatype such as frame play rate. In our practical work we have concentrated on end system resource capabilities since network aspects have been the subject of much attention in other studies.

\section{GENERAL PRINCIPLES}

\subsection{System Description}

The QoS Configuration System (Figure 1) provides a method for configuring services which is largely generic, requiring a minimal amount of service specific logic. It is a distributed application consisting of two parts:

- The Configuration Engine, which is located on a single machine.

- A number of Terminal Resource Agents (TRA), one on each computer that is participating in a service.

Each end-system is fully involved in the configuration of a service by providing resource configuration information to a service, satisfying the principle of end-toend systems design[2]. 


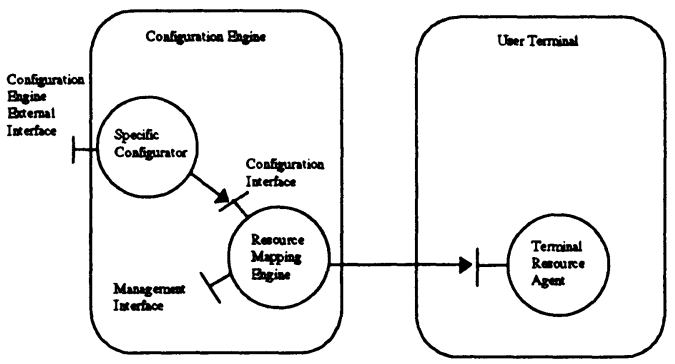

Figure 1 QoS Configuration System Overview

\subsection{QoS Profiles}

We have used the concept of QoS profiles. We have four types of profile (Figure 2): user, terminal, media, network, as derived from the different viewpoints on a service.

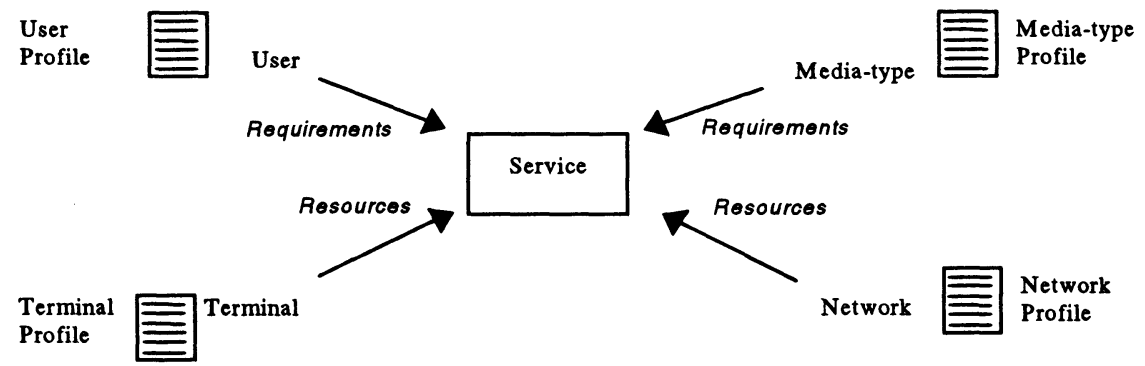

Figure 2 Viewpoints on a Service

These profiles represent a declarative approach to stating the QoS requirements of a service and the system resources available to fulfil them. We have found that it is not possible to configure a service in a purely declarative manner, and that there must be some specific procedural logic to fully configure a service.

In our system we have only implemented the media-type and terminal profiles, which are stored in the Configuration Engine and the TRA respectively. Each TRA, representing each terminal involved in the service session, is asked to compare its profile with the media-type profile and say whether the terminal is capable of supporting that media-type.

\section{IMPLEMENTATION AND EXPERIENCES}

Our practical work is based upon a video/audio-conferencing system. The conference organiser has the choice of either creating an audio/visual Mbone conference using the vic and vat tools or making a CU-SeeMe call directly between themselves and another user $[3,4,5]$. In an ideal system this choice would be invisible to the user; a multimedia conferencing system would select the best 
application(s) for the task. However, this would require an additional negotiation layer between the Terminal Resource Agents and the Configuration Engine.

\subsection{Using the system}

Through the use of an almost obligatory WWW interface, the conference organiser chooses the conference participants and the desired media-types. The list of participants in the service and the service required are passed to the Configuration Engine. The Configuration Engine then maps the list of participants to the terminals they are currently using. The profiles of the mediatypes needed to run the service are retrieved from a database. The Configuration Engine then forwards the media-type profile(s) to each Terminal Resource Agent. This checks the media-type profile with its terminal profile and returns one of the following results:

- The terminal is fully capable of supporting the media-type.

- The terminal is NOT capable of supporting the media-type.

- The terminal is not CURRENTLY capable of supporting the mediatype but is potentially able to do so.

The Configuration Engine then configures the service according to the results obtained.

Applications are individually configured for each user participating in the service, again with a WWW interface being used to launch helper applications.

\subsection{Alternative Styles of Working}

There are actually two methods in which the profiles can be passed:

- Media-type profile passed to TRA (resources requested), simple result returned.

- Simple request to TRA made, terminal profile returned (resources offered).

In the case of video-conferencing with a large number of users it is more scalable for the media-type profiles to be sent to each Terminal Resource Agent. Thus the comparisons can be conducted in parallel and the load is distributed over a number of machines.

There is also a choice as to how the TRA obtains the information for the terminal profile. The two choices are as follows:

- Agent periodically polls the system seeking an update on resources.

- Agent makes system calls to discover resources when requested.

In our implementation we have chosen the latter course of action, with the TRA obtaining its terminal profile through a system call to the Windows 95/Windows NT registry. 


\section{FUTURE EXTENSIONS}

We have already mentioned in section 2 above, that there are 4 separate profiles (terminal, media-type, network and user) but only the first two of these have been implemented. Further work will extend to cover the other profiles.

The system could also be used to configure adaptive services. Take as an example a user moving from an office environment to a mobile one. In the office they will have access to a high performance terminal and a high bandwidth connection. This changes to a lower performance terminal operating over a restricted bandwidth. The QoS Configuration System will be used to determine which media-types can be run on each terminal. On the former the user might be able to participate in a video and audio conference. On the latter, the user would participate in the same conference but with only an audio link.

\section{CONCLUSIONS}

The QoS Configuration System offers the following benefits:

- Abstraction over heterogeneous networks, applications and end systems. The Terminal Resource Agents all have the same interface regardless of the system configuration. All applications are dealt with in the same way with only a small amount of service dependent logic.

- Predictable session instantiation.

- Simple user controls/interface. The user only has to state what they want set up, not how it is to be done.

- Cost reduction to the provider of the service.

New applications can be introduced by producing new media-type profiles; there is no need to change the Terminal Resource Agents. Also, the requirements of a media-type can be changed without having to change either the Terminal Resource Agents or the specific service logic. The interface of the terminal agent abstracts over the underlying terminal.

\section{REFERENCES}

[1] K Nahrstedt J M Smith. The QoS Broker. IEEE Multimedia, Spring 1995 [2] J H Saltzer, D P Reed, D D Clark. End-to-End arguments in system design. ACM Transactions on Computer Systems. Vol 2 No 4. Nov 1984

[3] H Eriksson. Mbone: The Multicast backbone. Communications of the ACM, Vol. 37, No. 8. Aug 94.

[4] vic: A Flexible Framework for Packet Video. S McCanne and V Jacobson. Proc. ACM Multimedia, San Francisco, CA. Nov 1995

[5] Cornell University CU-SeeMe. http://cu-seeme.cornell.edu/ 